\title{
Activités
}

8-1 | avril 2011

Varia

\section{Tension entre indicateurs de production et indicateurs de sécurité}

Le cas de la certification d'une entreprise tunisienne

Tension between production indicators and safety indicators : case of the

certification of tunisian firm

Catherine Fournier, Raouf Ghram, Tahar-Hakim Benchekroun et Francis

Six

\section{(2) OpenEdition}

\section{Journals}

Édition électronique

URL : http://journals.openedition.org/activites/2457

DOI : 10.4000/activites.2457

ISSN : $1765-2723$

Éditeur

ARPACT - Association Recherches et Pratiques sur les ACTivités

\section{Référence électronique}

Catherine Fournier, Raouf Ghram, Tahar-Hakim Benchekroun et Francis Six, « Tension entre indicateurs de production et indicateurs de sécurité », Activités [En ligne], 8-1 | avril 2011, mis en ligne le 15 avril 2011, consulté le 06 mai 2019. URL : http://journals.openedition.org/activites/2457 ; DOI : 10.4000 /activites.2457

\section{(c) $(1)$}

Activités est mis à disposition selon les termes de la licence Creative Commons Attribution - Pas d'Utilisation Commerciale - Pas de Modification 4.0 International. 


\title{
Tension entre indicateurs de production et indicateurs de sécurité :
}

\section{Le cas de la certification d'une entreprise tunisienne}

\author{
Catherine Fournier \\ Laboratoire PSITEC (Psychologie: Interaction, Temps, Emotions, Cognition), EA4072, Université Charles-de-Gaulle, \\ Lille 3, BP 60149, 59653 Villeneuve d'Ascq Cedex, Catherine_fournier20002000@yahoo.fr \\ Raouf Ghram \\ Laboratoire PSITEC - Université Charles-de-Gaulle - Lille 3

\section{Tahar-Hakim Benchekroun} \\ Conservatoire National des Arts et Métiers, Centre de Recherche sur le Travail et Développement, 41, rue Gay-Lussac, \\ 75005 Paris

\section{Francis Six} \\ Laboratoire PSITEC - Université Charles-de-Gaulle - Lille 3
}

\begin{abstract}
Tension between production indicators and safety indicators: case of the certification of tunisian firm. Companies' chief sources of concern are related to profitability issues. Most decide to obtain certification in order to maintain their operations, and this requires them to use indicators. Some research has shown how this approach can cause safety issues to be "forgotten".

Our research took place in a firm based in Tunisia which in 2004 introduced a quality management system that resulted in certification. This was undertaken to comply with customer expectations and to keep the company in business.

We will show here how the use of indicators can sometimes cause safety issues to be pushed aside. The problem of a gap between what the indicator shows about the business and what is actually happening in the workplace leads to decisions being taken that do not result in the improvement of working conditions.
\end{abstract}

KEYS-WORDS

management indicators, certification, security, compromises, activity

\section{Introduction}

L'objectif de cet article est d'exposer en quoi et comment une démarche de certification et l'usage des indicateurs qui en découle peuvent avoir un impact sur la façon dont sont gérées les questions de santé et de sécurité au travail. Nous montrerons ici en quoi un indicateur de gestion de production masque les compromis faits par les opérateurs pour atteindre les objectifs de production, ces compromis se faisant parfois au détriment de leur propre sécurité. Nous regarderons plus spécifiquement la façon dont l'indicateur est utilisé par les différents niveaux hiérarchiques et en quoi cette utilisation 
est dépendante des contraintes de leur mission et de leurs représentations des réalités de la production et des activités individuelles et collectives mises en œuvre pour atteindre les objectifs en qualité et en quantité ainsi que de la sécurité.

La question se pose alors de voir comment sont abordées les questions de la sécurité au travail dans une entreprise qui a mis en place un système de management de la qualité (SMQ) d'autant plus que le lien entre santé/sécurité et travail, en terme de reconnaissance par la société, est assez récent (Coutarel, Daniellou, \& Dugué, 2005). Cette entreprise cherche à diminuer le nombre d'Accident du Travail (AT) d'une part pour diminuer les coûts de la sécurité et d'autre part parce que le groupe étranger auquel elle appartient en partie fait pression pour que ces questions soient traitées. L'entreprise appartient à la fois à un groupe tunisien et à un groupe étranger de fabrication de produits manufacturiers; elle est basée en Tunisie. Cette entreprise a mis en œuvre depuis 2004 un système de management de la qualité qui a débouché sur une certification ISO 9001 2000. La démarche a été initiée pour répondre aux demandes des clients et pour pérenniser l'activité de l'entreprise. Cette entreprise connaît par ailleurs des AT jugés trop nombreux et souhaite avoir un diagnostic de la situation pour mieux en comprendre les causes, diminuer leur survenue et de ce fait en réduire les coûts.

Dans cet article, nous allons dans un premier temps faire un point bibliographique sur la certification, ses apports et ses limites. Nous ferons également le lien entre certification et activité des opérateurs. Ensuite, nous discuterons de la notion de risques. Enfin, nous décrirons les situations de travail rencontrées et les compromis faits par les opérateurs pour faire face aux exigences de la tâche avant de revenir sur les indicateurs de gestion. Nous conclurons sur les apports possibles de l'ergonomie à cette problématique.

\section{1.- Un point sur la certification}

La préoccupation principale des entreprises est la rentabilité. Pour s'assurer de celle-ci et pour répondre à des critères commerciaux, elles mettent en place des Systèmes de Management de la Qualité (SMQ) qui aboutissent généralement à une certification. Cette démarche a surtout connu son essor dans les années 1990 et a renouvelé les pratiques de production, de gestion et de management (Ohno, 1989, cité par Iazykoff, 2004, p. 2).

Fin décembre 2004 on comptait plus de 27101 entreprises certifiées ISO 9001 en France, 326895 en Europe et 670399 dans le monde. Les chiffres de la Tunisie ne sont pas communiqués mais on sait par ailleurs que ces démarches y sont de plus en plus mises en place.

\section{1.- Quelques définitions}

La certification peut être définie selon plusieurs dimensions (Mambi-El-Sendegele, 2001):

- c'est un instrument de réduction de la complexité; cette complexité est due en partie aux relations qu'entretient une entreprise avec une multitude de fournisseurs, dont la qualité de la production est variable. Il peut donc exister un écart plus ou moins important entre ce qui est prévu au marché et attendu et ce qui est effectivement livré. C'est ce que Mambi-El-Sendegele (2001) appelle la contingence. La norme, par le contrat qu'elle oblige de passer entre les fournisseurs et l'entreprise va permettre de diminuer à la fois la complexité et la contingence. Une négociation bilatérale entre les deux partenaires se fait alors sur la qualité ;

- c'est un dispositif cognitif institué; il s'agit pour les différents partenaires de s'accorder sur des conventions, c'est-à-dire sur la régularité des comportements à adopter comme une sorte de référentiel commun ;

- c'est un argumentaire d'échange industriel; il s'agit pour l'entreprise de valoriser le niveau de ses interventions et de ses prestations. De plus, cela constitue pour les clients un critère de sélection. Enfin, cela régule le marché. 
La norme qui nous intéresse ici particulièrement est la «norme qualité », qui conduit à la mise en place dans les organisations de SMQ. La qualité y est définie comme une «aptitude d'un ensemble de caractéristiques intrinsèques à satisfaire des exigences » (AFNOR, 2001, p. 22).

La norme fixe des exigences mais il est libre à l'entreprise de définir ce qu'elle entend par qualité du produit. Cependant, l'entreprise, pour mettre en place un SMQ, doit « démontrer son aptitude à fournir régulièrement un produit conforme aux exigences des clients et aux exigences réglementaires applicables » et doit viser à « accroître la satisfaction de ses clients par l'application efficace du système $[. .$.$] » (cf. Normes NF EN ISO 9001). La qualité va donc être définie par l'entreprise elle-même$ en fonction des exigences réglementaires, celles du marché et des clients.

Les notions de certification et de qualité sont étroitement liées. Ces liens sont dus en partie à leur évolution conjointe du fait des transformations du contexte socio-économique. La qualité possède un « pouvoir structurant, des propriétés pérennisantes et une capacité à susciter une dynamique d'adoption » (Penan, 1995 et Reynaud, 1988, cités par Lérat-Pytlak, 2002, p. 44). Ces caractéristiques apparaissent comme le levier qui a permis son développement pour en faire un critère de management de la normalisation. La certification (ou normalisation) et la qualité ont d'abord concerné le produit, puis les processus industriels et organisationnels pour aujourd'hui être intégrées dans le management et les stratégies de l'entreprise. Cette évolution peut s'expliquer par le fait que le nombre toujours croissant d'entreprises certifiées tend à diminuer l'enjeu commercial et pousse les organisations à aller toujours plus loin dans la démarche.

De plus, la notion de qualité est une notion floue et pourtant très répandue. Au-delà de sa définition normalisée, la qualité constitue plus une construction sociale propre aux accords et compromis faits dans chaque entreprise concernée (Laurens, 1997). Elle est généralement subjective, en fonction de l'individu ou de l'entité qui la définit, mais aussi du corps de métier auquel il appartient. Il y a donc des qualités. On peut remarquer également que généralement la qualité n'est pas définie dans les entreprises qui mettent en place un SMQ (Mispelblom, 1995). Celle-ci est en fait perçue comme tellement évidente qu'il ne sert à rien de la formaliser. De ce fait, elle va relever davantage d'un jugement de valeur de ce qu'est un bon produit ou un bon travail. Il nous apparaît pertinent de préciser ceci car c'est précisément le phénomène que l'on retrouve dans l'entreprise qui fait l'objet de cette recherche: en effet, la qualité n'est pas définie de façon formelle, mais relève plus d'une appréciation. Ce constat ressort des entretiens menés avec trois membres du comité de direction (soit le directeur «pôle gestion », le directeur Recherche et Développement et le directeur technique), en vue de connaître leur point de vue et leur implication dans la mise en place de la démarche qualité. Pour ces trois personnes, la qualité du produit et des services rendus est avant tout définie par rapport aux besoins des clients et est donc dynamique et non stabilisée. Pour arriver à obtenir la qualité souhaitée, il faut que le personnel «pense qualité » (cité par le directeur technique) et ait la «mentalité qualité » (cité par le directeur recherche et développement) même si cela peut paraitre lourd et procédurier (d'après le directeur pôle gestion).

\section{2.- Les effets de la certification}

Les principales motivations des entreprises à la mise en place des SMQ sont la pression des clients recherchant des assurances sur la qualité des produits, de la concurrence et la volonté de décrocher de nouveaux marchés. Cela suppose qu'il faille fiabiliser les processus de production et maîtriser les coûts.

Cependant, la certification place l'entreprise dans des situations paradoxales, des dilemmes (LératPytlak, 2002):

- le dilemme entre les besoins des clients et les exigences spécifiées : la transcription des besoins explicites ou non des clients peut poser de grandes difficultés aux entreprises. En effet, le but de la norme ISO n'est pas de garantir un certain niveau de performance mais de montrer au 
client que son prestataire a mis en place une organisation qui veille à satisfaire ses besoins et qui cherche à s'améliorer. La formalisation d'un contrat entre les deux parties est donc rendue difficile ;

- le dilemme entre les procédures standardisées et l'autonomie des acteurs: la littérature est assez riche concernant l'inadéquation entre d'une part la formalisation d'un prescrit que les opérateurs doivent suivre et les exigences réelles des situations de travail auxquelles ils ne peuvent faire face qu'à condition qu'ils aient une certaine autonomie ou pouvoir d'agir ;

- le dilemme entre les procédures standardisées et l'appropriation par les acteurs : «l'efficacité de la règle formelle tient au fait que ceux qui doivent la mettre en œuvre se l'approprient, l'adaptent ou la redéfinissent et non pas à son énoncé qui comporte des blancs ou des "trous" 》 (de Terssac, 1994). L'efficacité des procédures réside donc en partie dans la façon dont elles ont été conçues, dans la façon dont le personnel a été sollicité pour cela. Cependant, les procédures sont transgressées pour deux raisons: parce qu'elles sont forcément en inadéquation avec la réalité (en tout cas à certains moments de l'activité) et parce que les opérateurs ont besoin de disposer de marges de manœuvre pour permettre au système de fonctionner (Bourrier, 2005).

Les effets de la mise en place de SMQ et de certification qualité ont été étudiés en ergonomie et en sciences de la gestion. Les résultats sont assez mitigés, notamment en ce qui concerne les impacts sur la santé et la sécurité.

Les premières critiques portent sur le rapprochement que font certains auteurs entre certification et organisation taylorienne. Ces deux types de management ont en effet des points communs du fait du recours aux procédures formalisées et aux contrôles réguliers tels que les audits (Iazykoff, 2004). Dans d'autres approches, on va considérer qu'au contraire la certification permet une appropriation de la démarche plus importante que dans le cas du taylorisme car il s'agit d'une conception "indigène » de la démarche et des procédures (Iazykoff, 2004, p. 3). Pourtant, comme le précisent Sauvagnac et Falzon (2000), la mise en place de procédures est rarement établie en fonction des savoirs déjà acquis par les opérateurs. De plus, elle n'est mise en place que de façon descendante. Elle est alors généralement perçue comme une prescription de plus (Campinos-Dubernet \& Jougleux, 2003).

L'instauration de la démarche qualité dépend de l'organisation déjà en place, elle ne la modifie pas mais la rend plus lisible. Ce système de gestion documentaire peut être également perçu comme «un alourdissement des ajustements progressifs qui peuvent être entrepris au fur et à mesure des avancées cognitives »(Campinos-Dubernet \& Marquette, 1997, p. 43). Cependant, les salariés perçoivent dans certains cas ce système comme une source d'autonomie.

Il permet ainsi de coordonner les actions notamment lors de procédures de résolution de problèmes (par exemple des documents standard sont utilisés, les communications d'informations se font par le même support). Ces procédures ou dispositifs sont admis par tous. La «norme qualité » a donc un but informationnel et peut être qualifiée de «dispositifs cognitifs collectifs» (Lerat-Pytlak, 2002).

La question des SMQ a aussi été étudiée selon ses impacts sur les questions de santé et sécurité au travail (SST). Notamment, une étude québécoise (Dionne-Proulx, Carrière, \& Beauchamp, 2005) a montré qu'il est rare que les entreprises intègrent la question des SST lors de la mise en place d'un SMQ, d'une part parce qu'elles peuvent manquer de ressources pour le faire et d'autre part parce qu'elles estiment que la certification ne laisse pas d'espace pour ces questions. Le manque de visibilité concernant les liens entre qualité et SST varie en fonction de la taille des entreprises, plus l'entreprise est grande plus elle perçoit ces liens. Cette étude montre également qu'il n'y a pas d'impact de la mise en place d'une certification qualité sur la SST.

On peut toutefois dire que la sécurité est généralement pensée en termes techniques et sur du court terme. Par exemple, la nécessité d'intégrer quelques aspects de sécurité peut découler des normes imposées relatives à la circulation dans les allées, à l'utilisation et au stockage de matériels, etc. (Cheikh, 2000) ce qui est cohérent avec l'approche technico-réglementaire largement répandue de la prévention des risques (Garrigou, Peeters, Jackson, Sagory, \& Carballeda, 2004 ; Six, 1999). 


\section{3.- Certification, indicateurs de gestion et activité}

Les indicateurs de gestion servent à investiguer les fonctionnements organisationnels, à piloter le changement et à explorer les situations nouvelles. On les retrouve dans tous les secteurs de l'entreprise (modèles statistiques de prévision des ventes, résultats des périodes précédentes, coûts de fabrication logistique, prix de revient...) (Moisdon, 1997).

Un indicateur est « une information devant aider un acteur, individuel ou plus généralement collectif, à conduire le cours d'une action vers l'atteinte d'un objectif ou devant lui permettre d'en évaluer les résultats $\gg(L o r i n o, 2003$, p. 131). Il peut prendre diverses formes (chiffres, repères qualitatifs...). Nous pouvons également ajouter à cette définition qu'un indicateur résulte d'un «petit nombre d'informations qui ont la propriété d'être particulièrement signifiantes par rapport aux objectifs stratégiques de l'entreprise » (Savall \& Zardet, 2003, p. 85).

Un indicateur contient plusieurs informations (Lorino, 2003): l'objectif stratégique auquel il est attaché; l'acteur le produisant; le responsable de la performance représentée; la fréquence avec laquelle il est produit et suivi; la façon dont on l'obtient (formule de calcul, sources d'informations utilisées...) ; les modes de segmentation; la présentation ; la liste de diffusion.

Dans une organisation, on peut aussi trouver des indicateurs de santé (maladies professionnelles déclarées, accidents du travail déclarés...), de gestion du personnel (effectif, intérimaires, heures supplémentaires...), de gestion de production par les opérateurs (longueur des séries, durée des arrêts machine...), d'organisation du travail (nombre de postes tenus, fréquence des rotations...) (Bourgeois, Lemarchand, Hubault, Brun, Polin, \& Faucheux, 2000).

Lorsque ces indicateurs sont censés rendre compte des conditions de travail, ils ne vont souvent révéler qu'une combinaison de facteurs, résultat d'une approche unicausale alors qu'une approche pluridisciplinaire serait nécessaire (Leplat, 1997).

Comme nous l'avons dit précédemment, la normalisation sous-entend la mise en place de procédures et de processus formalisés. Au départ, les démarches ISO obligeaient à la mise en place de procédures figées et rigides. La version de la norme ISO 9001 « $2000 »$ n'utilise plus ces schémas d'organisation mais a ouvert ses prérogatives à un modèle plus souple et plus ouvert appelé « approche processus ». Ce type d'approche, que nous allons définir ci-après, laisse plus de marges de manœuvre aux entreprises puisque ce sont elles-mêmes qui définissent les processus dont elles ont besoin en mettant des liens entre les activités principales. Il s'agit de « gestion de la coopération d'activités qui ne se caractérisent pas par leur similitude, mais par leur complémentarité pour atteindre un certain type de résultat, porteur de sens au plan technique, économique ou social » (Lorino, 2009).

Un processus peut être défini comme un «enchaînement d'activités ou d'ensembles d'activités, qui est alimenté par des entrées, qui dispose des ressources et qui ajoute de la valeur par rapport au but pour créer des sorties. Les entrées d'un processus proviennent soit de l'extérieur, soit d'un autre processus (processus amont). Tout comme ses sorties vont soit vers l'extérieur, soit vers un processus aval » (Brandenburg \& Wojtyna, 2006, p. 51). Les processus doivent pouvoir être mesurés pour contrôler les entrées et les sorties (efficacité), pour piloter leurs performances (efficience), pour déterminer leur maturité (excellence), et leur valeur. Ils doivent être stables et reproductibles.

Les termes ici utilisés le sont également en ergonomie mais parfois avec quelques différences que nous allons préciser; ainsi en ergonomie:

- on parle de tâche et d'activité (Guérin, Laville, Daniellou, Duraffourg, \& Kerguelen, 2001); en gestion, seul le terme d'activités est utilisé et réfère plutôt à ce qui est appelé en ergonomie la tâche (Savall \& Zardet, 2003);

- on considère que la performance c'est à la fois l'efficacité (atteinte ou non des résultats) et l'efficience (moyens mis en œuvre pour y arriver) (Bourgeois \& Hubault, 2005); de ce fait, l'ergonomie s'intéresse à la fois à la performance économique et humaine et cherche à les 
confronter entre elles (Six, 1999); en gestion, il s'agit de ce qui permet d'une part d'améliorer le couple valeur-coût et d'autre part d'atteindre les objectifs stratégiques (Lorino, 2003). Les gestionnaires représentent l'articulation entre les objectifs, les ressources et les résultats attendus sous forme de triangle:

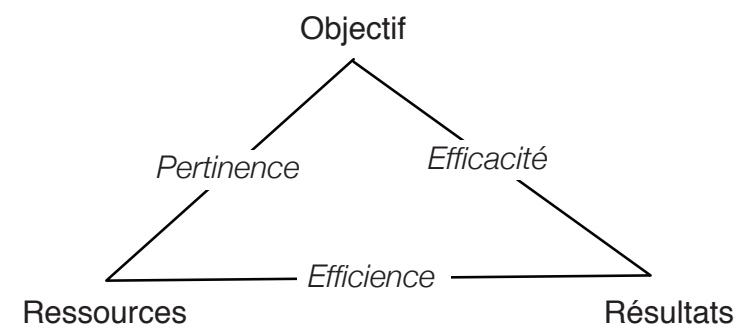

Schéma 1 : triangle de la performance (Hubault, 1998)

Figure 1 : Performance triangle (Hubault, 1998)

La pertinence symbolise ici la cohérence entre les objectifs poursuivis et les ressources mises en place à cette fin, il s'agit de la représentation de la consommation de ressources d'une entreprise pour l'atteinte d'un but. L'efficience se caractérise par la contribution des ressources à l'atteinte des résultats. L'efficacité rend compte de la correspondance entre les objectifs et les résultats. On peut donc retenir qu'une entreprise peut être à la fois efficace (objectifs = résultats) sans être efficiente (ressources insuffisantes ou au contraire consommation trop importante de ressources) et inversement. De plus, l'efficacité et l'efficience ne peuvent s'analyser sans regarder la pertinence (Hubault, 1998). En outre, on constate que dans les études des gestionnaires sur la performance, les coûts cachés de la santé sont rarement pris en compte. Ainsi, dans la première définition des gestionnaires, il est plutôt question d'efficacité que de performance. Or les indicateurs de gestion sont construits par des gestionnaires selon cette définition. Nous posons donc la question de savoir si les indicateurs de gestion ne seraient pas simplement révélateurs de l'efficacité d'un processus, c'est-à-dire qu'ils ne rendent pas compte de ce qui est mis en jeu par les différents acteurs de l'entreprise, en particulier les opérateurs, pour atteindre les résultats.

La recherche présentée étant bien une recherche en ergonomie, nous retiendrons ici les définitions propres au champ conceptuel de cette discipline. Cependant, il est important de comprendre les définitions des gestionnaires pour pouvoir interpréter et comprendre leur recherche et voir en quoi les notions abordées diffèrent ou se rapprochent du sens qu'elles ont en ergonomie.

Il est à noter qu'au-delà de l'indicateur lui-même, il faut regarder ce que les opérateurs en font en fonction des déterminants de leur activité. Dans la mesure où un indicateur de résultat est comparé à un objectif préfixé, il joue comme une contrainte de l'activité de ceux qui doivent s'y référer, c'est le cas de l'encadrement de proximité qui a en charge le suivi de la production. En effet, la direction d'une entreprise doit prendre des décisions de façon rapide, elle a donc recours à des informations pratiques à interpréter et « règle logiquement ses choix de manière à optimiser les jugements dont elle [il] se sent l'objet» (Berry, 1983, p. 9). D'ailleurs, certaines études du Centre de Recherche en Gestion (CRG) en France montrent que plus on monte dans la hiérarchie plus il semble difficile de quantifier les problèmes pour les résoudre. Un indicateur a une nature subjective (Lorino, 2003), en effet, le lien entre l'indicateur choisi et l'objectif fixé est indirect. C'est après avoir choisi l'action pour atteindre l'objectif qu' on choisit l'indicateur. De plus, l'indicateur se construit et est une mesure émanant de l'interprétation de la réalité (Moisdon, 1997). 
Cependant, le recours aux indicateurs pose un certain nombre de questions. Leur utilisation résulte souvent d'une « rationalité éclatée » (Berry, 1983, p. 14); les différents acteurs utilisant des indicateurs, parfois les mêmes, ne peuvent pas prendre en compte, pour la prise de décision, toutes les logiques de tous les autres acteurs de l'organisation. En effet, les informations à prendre en compte seraient trop nombreuses et le confinement parfois obligatoire ce qui peut engendrer des contradictions entre les différentes actions menées (Berry, 1983; Moisdon, 1997).

Dans la problématique qui nous intéresse ici, nous ferons dans un premier temps le constat des limites intrinsèques d'un indicateur de production mais surtout montrerons en quoi il est important de regarder la façon dont cet indicateur est utilisé en articulation avec les contraintes des utilisateurs.

\section{2.- Gestion du risque et compromis}

La demande de l'entreprise qui fait l'objet de cette intervention portait sur les AT et leurs causes. Nos observations nous ont conduits à identifier des situations à risques que nous présenterons plus loin après un point bibliographique sur la notion de risque.

Le risque et sa gestion sont des notions étudiées depuis les années 70. Ils sont rarement étudiés seuls mais souvent en lien avec la notion d'erreur et de défaillance. De nombreuses disciplines s'y sont intéressées (psychologie, ergonomie, sociologie...). Le point commun de ces différentes approches est basé sur trois principes (Amalberti, 2004) : une entreprise ne peut tolérer l'erreur car cela perturbe son activité; puisque le «zéro accident » ne peut être atteint, il faut tout de même minimiser le risque au maximum; les violations de règles sont inadmissibles. En outre, la notion d'erreur varie selon que l'on s'intéresse aux processus cognitifs, à la qualité ou à la sécurité des systèmes.

Les travaux en ergonomie soulignent la nécessité d'élargir le champ d'étude de la situation de travail à celle de la stratégie de l'entreprise et aux environnements technique et social (De La Garza, 2004). En effet, l'explication, grâce à l'analyse de l'activité, des dysfonctionnements aboutissant à des AT n'étant pas suffisante pour les éviter, des questions vont se poser au niveau de la conception de ces environnements. Ainsi, on a pu identifier des types d'erreurs (par exemple, Reason, 1993) et leurs causes reconnues comme dépendantes de sphères hiérarchiques bien plus élevées que celles où ont lieu les AT. Les nouveaux modèles tentent d'adopter une approche systémique et dynamique des situations de travail.

Le risque est une notion abstraite que l'on peut déduire des observations «concernant les techniques mises en cuvre, l'environnement de travail, les comportements des opérateurs en situation » (Six, 1999, p. 70). Il peut être défini en terme de probabilité d'occurrence d'un événement non souhaité et de gravité des effets produits. Sa gestion consiste alors à diminuer cette probabilité mais aussi son coût (Hoc, 2004). En fonction des points de vue, il peut être considéré comme une mesure du niveau de danger. Son évaluation résulte de la création d'un modèle propre aux théories des professionnels qui l'utilisent (Dos Santos, 2002, cité par Garrigou, Peeters, Jackson, Sagory, \& Carballeda, 2004, p. 499). Il peut être géré au niveau individuel et collectif.

La gestion du risque par l'individu laisse entendre que celui-ci ait conscience du risque, dans ce cas nous parlerons de prise de risque. Goguelin (2003) a ainsi posé que quatre conditions étaient nécessaires pour pouvoir parler de prise de risque: (1) le sujet doit être placé dans une situation dans laquelle il doit agir; (2) l'action envisagée doit comporter une probabilité d'échec entraînant des dommages pour lui ou pour les autres; (3) le sujet doit être conscient du risque, il peut en estimer la gravité et la probabilité; (4) une fois qu'il décide de la mettre en œuvre, son action et ses conséquences ne pourront plus être arrêtées. La prise de risque doit être replacée dans un contexte où généralement elle est interdite formellement par des procédures prescrites. Ces procédures sont d'autant plus présentes que l'entreprise est soumise à des normes. Ces procédures ou règles de sécurité peuvent être appliquées ou non par le sujet en fonction de leur acceptabilité et de leur accessibilité 
(Leplat, 1998). Pour autant, dans le même article, Leplat (1998) conclut à la nécessité de ces règles. La question de leur construction (par qui, comment et pourquoi ?) se pose alors. En effet, si ces règles sont perçues comme «illogiques, ou trop contraignantes [...] évaluées comme cô̂teuses ou comme non pertinentes par rapport à leur objet $\gg$ (Forrierre, 2008, p. 58), elles ne seront pas mises en œuvre ou respectées par les opérateurs. Une étude de Bourrier (2005) comparant deux façons de créer et de modifier les procédures montre que pour que celles-ci soient mieux acceptées et respectées, il faut réunir trois « ingrédients » : la possibilité d'un feed-back des opérateurs vers leur hiérarchie, des ajustements venant directement des personnes concernées par les procédures et une mise en place rapide.

Trois axes peuvent être envisagés pour diminuer les risques (Amalberti, 2004): (1) la qualité par le suivi des procédures et mesurée par les indicateurs; (2) la suppression du risque à sa source en supprimant le danger; (3) la construction de défenses pour récupérer la défaillance. C'est principalement le premier axe qui est retenu dans le cadre de la mise en place de normes.

Dans le cas de la certification, comme nous l'avons déjà mentionné plus haut, nous savons que les procédures, les règles de sécurité y compris, sont rarement rédigées par rapport aux compétences et savoir-faire des opérateurs (Sauvagnac \& Falzon, 2000). Or, on sait que les savoir-faire des opérateurs relèvent parfois de compromis faits pour atteindre les objectifs de production même au détriment de leur santé. C'est ainsi que l'on considère les comportements dans le travail (comme par exemple la prise de risque) comme «l'expression du coût, pour l'opérateur, de la gestion des compromis manifestée dans l'activité » (Teiger, 1993). Cela peut entraîner une violation des règles, ce que nous illustrerons dans la partie consacrée aux résultats.

\section{1.- Résultats de l'Analyse du Travail}

Comme nous l'avons dit en introduction, la recherche porte sur une entreprise située en Tunisie. Cette entreprise a mis en œuvre depuis 2004 un système de management de la qualité qui a débouché sur une certification ISO 90012000 afin de satisfaire aux exigences de performance et de rentabilité de plus en plus exigées par le marché économique mondial.

En effet, comme beaucoup de pays en voie de développement, la Tunisie a bénéficié dans les années 1970 des délocalisations des entreprises du « Nord » (Ben Hammouda, 1995) grâce au faible coût de sa main-d'œuvre. Pour garder ces entreprises sur son sol, le gouvernement tunisien a alors mis en place des aides et autres avantages dans les années 1980 (accord de libre-échange avec l'Union Européenne, exonération fiscale, rapatriement des bénéfices...). Géographiquement située au carrefour de deux continents (Afrique et Europe avec une ouverture sur le Proche et Moyen Orient), la Tunisie continue d'intéresser les investisseurs (Sfeir, 2006). Pourtant, la concurrence notamment de la Chine, se fait sentir. De ce fait, les entreprises implantées principalement sur les zones côtières se préoccupent de plus en plus de leur performance et de l'évaluation de celle-ci. C'est ainsi que nous retrouvons ici la même volonté que dans les pays du Nord de gérer les coûts pour être le plus compétitif possible. Les questions de santé et de sécurité (et de leurs coûts) deviennent également importantes, dans un contexte où pérenniser la présence des entreprises et des investisseurs devient crucial (Benchekroun, Ghram, Fournier, Six, \& Akrout, 2009).

Dans cette recherche, nous avons mis en place une démarche d'intervention basée sur la méthodologie de l'analyse ergonomique du travail. Nous avons également utilisé la méthodologie de l'analyse des traces d'un indicateur de gestion de production appelé Feuilles d'équipes (FE) sur lesquelles sont reportés les taux de rebuts, le nombre de pièces produites et surtout les temps d'arrêt machine, leurs causes et les interventions faites pour réparer les pannes. Nous avons cherché par cette analyse des éléments expliquant les situations à risque et leurs déterminants identifiés par les observations. Ces deux approches ont ensuite été comparées pour analyser à la fois ce qui ressort des FE et qui sert à la prise de décision et les déterminants de l'activité des opérateurs pouvant expliquer la survenue de situations à risque. 
Nous avons dans un premier temps effectué des observations ouvertes en parallèle d'entretiens pour comprendre l'activité et faire l'identification des différentes tâches réelles. Ces analyses ont porté sur le travail des opérateurs sur la chaîne en salle de production; dix observations ouvertes d'environ deux heures chacune ont été effectuées (cinq au poste du matin et cinq au poste de l'après-midi). Il s'agissait à chaque fois d'équipes différentes.

Dans la partie suivante, nous allons décrire les tâches réelles pour commencer à appréhender l'activité des opérateurs.

\section{2.- Description des tâches réelles et de l'activité des opérateurs}

L'entreprise comporte deux unités de production, dont l'unité « hygiène bébé » objet de cette recherche; les machines de production ont une moyenne d'âge de 12 ans. La diversité des produits se trouve au niveau de la taille des produits et de certaines caractéristiques physiques en fonction des marchés ciblés (par exemple le produit destiné à la France peut comporter des scratchs mais pas celui destiné à la Tunisie). Son effectif est de 98 opérateurs dont 13 membres de l'encadrement. Les équipes sont composées d'après les fiches de postes, d'un chef d'équipe, d'un conducteur de ligne et d'un aide conducteur, d'un contrôleur qualité sur ligne, d'un emballeur et d'un assistant de maintenance. Ce secteur comporte quatre lignes de production. L'unité de production fonctionne 24 heures sur 24. Le processus peut être considéré comme un processus automatisé de production séquentielle dans lequel de multiples composants sont insérés à différents endroits, c'est-à-dire des matières premières ayant subi des transformations; le produit fini en sortie est la couche-culotte. Les composants proviennent d'un ou deux fournisseurs au plus, la majorité d'entre eux (environ $80 \%$ ) étant des fournisseurs européens.

Sur ce processus, les tâches sont multiples et planifiées avec divers déroulements temporels et sont réparties entre les opérateurs en fonction de leur poste. Nous constatons que les opérateurs se remplacent sur les différents postes lorsque cela s'avère nécessaire ce qui constitue une certaine forme de polyvalence.

Les tâches réelles consistent à :

(1) approvisionner la machine en composants conditionnés sur des bobines: avant d'être insérées dans la machine, les bobines doivent être préparées; les bobines sont ensuite insérées dans la machine en fonction du dévidage de la bobine en cours; ces interventions ne nécessitent pas l'arrêt de la machine.

(2) gérer des pannes machines entraînant des arrêts: les arrêts peuvent avoir plusieurs causes: casse composants, dans ce cas, la machine s'arrête automatiquement du fait du "bourrage machine », l'opérateur doit alors nettoyer le site, passer manuellement le composant dans les roulements et remettre en marche la machine; casse machine: dans ce cas, il s'agit d'un élément technique de la machine qui casse ou se déforme du fait de l'usure, la machine s'arrête alors également automatiquement et l'opérateur doit réparer et effectuer la remise en marche; problème qualité : l'opérateur réalisant les contrôles remarque un défaut et le signale à ses collègues pour qu'ils effectuent un réglage.

Les deux premiers types d'arrêts (casse composants et casse machine) génèrent des arrêts automatiques. En revanche, les problèmes qualité sont gérés par les opérateurs sans arrêt machine pour éviter les pertes de temps de production. Les origines des pannes renvoient principalement à la qualité des composants et à la maintenance des machines. Par ailleurs, on observe des anticipations qui ont pour but de prévenir les arrêts machine dus aux casses composants dans les roulements en facilitant leur passage (ce qui évite les bourrages qui créeraient des arrêts automatiques par exemple) et des interventions pour régler la machine en cas de détection de défauts de qualité du produit.

Les arrêts peuvent donc être à l'initiative de l'opérateur ou être le fait des automatismes.

Les différentes étapes du processus peuvent être représentées ainsi: 


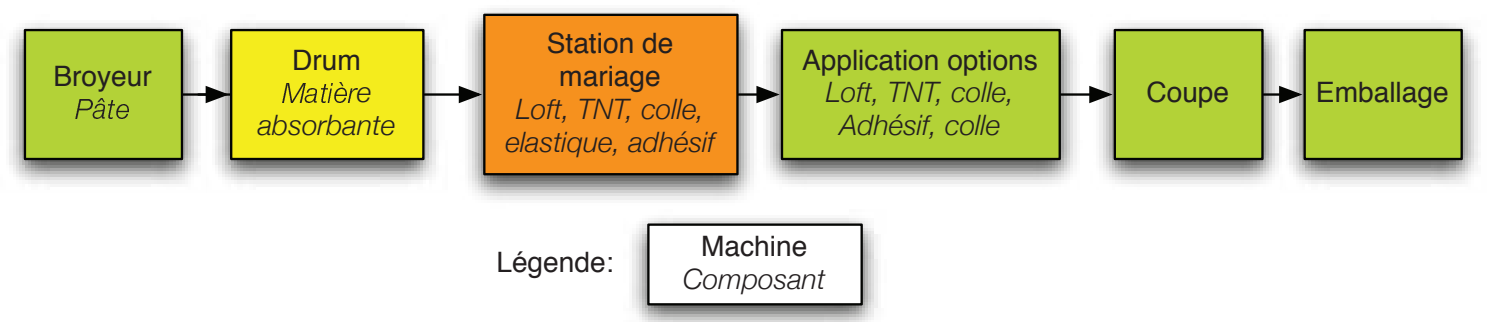

Figure 1. Processus de fabrication des couches-culottes

Figure 2: Manufacturing process for nappies.

La fabrication d'une couche-culotte se fait par ajouts successifs de composants, de un à cinq selon le sous-système. Nous avons pu identifier les lieux où les interventions sur machine en marche étaient les plus fréquentes (cadre sur fond orange), fréquentes (fond jaune), les moins fréquentes (fond vert) c'est-à-dire les lieux où les composants sont les plus sensibles. Ces interventions se font en fonction des types de composants insérés : par exemple le bloc «station de mariage » est davantage cause d'interventions non pas à cause du nombre de composants mais parce que les composants « loft » et «TNT » cassent très régulièrement au moment de leur arrivée dans le processus juste avant d'être insérés au niveau de la matière absorbante. En revanche, les composants du bloc « application options » n'ont pas ce type de défauts. Par ailleurs, ces composants subissent des tests avant d'être amenés dans le processus, tests qui portent uniquement sur les caractéristiques chimiques et non sur des caractéristiques physiques (élasticité, résistance...) relevant davantage du passage dans la machine. On observe donc des interventions sur machine en marche qui peuvent générer des situations à risque.

L'activité de gestion des pannes machine a donc une double fonction (Faverge, 1970) pour assurer le niveau de production: une fonction préventive, en minimisant le nombre d'arrêts par des actions sur machine en marche, et une fonction de récupération, lors des arrêts automatiques dus généralement à des problèmes techniques et mécaniques sur lesquels les opérateurs n'ont pas de prises directes.

Dans les deux cas, les opérateurs utilisent des informations formelles prélevées sur les instruments de mesure de la machine et surtout des informations informelles prélevées sur le composant directement. Ces opérations ne s'effectuent pas indépendamment les unes des autres. Les observations du processus par les opérateurs permettent d'anticiper les pannes, quelle que soit leur nature. En effet, les opérateurs peuvent savoir, en regardant et en écoutant le bruit des machines, que le composant est susceptible de casser, ou qu'un réglage est à faire. Ceci a bien sûr une limite, certaines pannes restent inévitables. Ces anticipations sont d'autant plus limitées que les opérateurs sont occupés à autre chose: par exemple, s'il y a un réglage à effectuer, l'opérateur ne peut plus observer le processus.

Les communications de l'encadrement de proximité avec les opérateurs de production se font à la fois de façon informelle mais également sur la base des FE. Nous reviendrons sur ces relations dans le paragraphe suivant.

\section{3.- La gestion du risque}

Aujourd'hui, dans l'entreprise, nous constatons un double discours: celui qui avant l'intervention mettait en cause l' « inconscience » des opérateurs quant à l'apparition des AT et celui qui depuis l'intervention ergonomique essaie de comprendre pourquoi il existe des violations de règles et des AT.

Deux points de vue sur la prise de risque peuvent être considérés :

- un point de vue extérieur où le risque est vu comme mal géré par les opérateurs et la prise de risque non justifiée ;

- un point de vue intérieur où les opérateurs ont conscience du risque mais ont développé des compétences permettant de faire face à ces situations et où la prise de risque se justifie par la 
volonté d'atteindre les objectifs, c'est ce que nous allons développer dans cette partie.

\subsection{1.- Le point de vue des opérateurs}

Les opérateurs ne sont pas toujours en capacité d'anticiper, en particulier lorsqu'ils sont occupés à des interventions sur machine. Dans ce cas des pannes surviennent entraînant des arrêts fréquents. Un certain taux d'arrêt ne permet pas l'atteinte de la production: c'est ce qui conduit les opérateurs à effectuer des interventions sur la machine alors que celle-ci est en fonctionnement. Pour cela, ils ont shunté les «switchs » des portes de sécurité. Ainsi, même lorsque les portes sont ouvertes, les switchs sont fermés avec de simples morceaux de carton et la machine ne s'arrête pas.

Les entretiens mentionnent l'existence d'un non-respect de la règle de sécurité concernant l'utilisation des switchs pour ouvrir les portes de sécurité. Les opérateurs expriment une certaine acceptation du risque, comme étant quelque chose d'inhérent au métier, à travers des expressions du type «on sait où mettre les doigts, et celui qui ne sait pas, quand il a un accident il recommence pas une deuxième fois ».

Toutefois, les opérateurs expliquent qu'il y a des limites aux interventions sur machine en marche: en effet, certains endroits ne font l'objet d'interventions que lorsque la machine est arrêtée car jugées trop dangereuses. De plus, on observe que les opérateurs mettent en œuvre des moyens de prévention tels qu'envelopper des parties de la machine de couches-culottes pour éviter les chocs. Ces savoir-faire de prudence participent à la gestion des risques en situation et sont guidés à la fois par des objectifs de performance et de réduction de la dangerosité des machines. Dans ce sens, la prise de risque est avant tout la résultante de compromis (personnel, individuel, organisationnel...) entre les ressources personnelles et les moyens à disposition (Garrigou, Peeters, Jackson, Sagory, \& Carballeda, 2004).

\subsection{2.- Le point de vue de la hiérarchie}

$\mathrm{Si}$ on observe des compromis tels que décrits chez les opérateurs, on en observe également chez l'encadrement de proximité, celui-ci a pour mission de faire respecter les règles de sécurité prescrites mais par ailleurs il connaît les situations de gestion des risques par les opérateurs. Seulement, il se trouve pris dans un étau entre d'une part ces compromis qui sont inadmissibles aux yeux de la prescription et de la ligne managériale et d'autre part les exigences de production.

Cette situation a été décrite dans d'autres secteurs d'activité, comme celui de la construction: l'encadrement est confronté à « un dilemme qui le conduit bien souvent à arbitrer entre deux positions délicates: soit «fermer les yeux » sur les entraves aux mesures de sécurité, sachant qu'elles demeurent le moyen d'atteindre les normes de rendement, soit se tenir à la prescription et la faire respecter, donc se mettre en situation de ne pas atteindre les résultats demandés... Cette dernière situation sera difficile à assumer par l'encadrement qui est, avant tout, chargé d'atteindre les objectifs de production » (Berthet, 1993, cité par Six, 1999, pp. 65-66). L'encadrement se trouve ainsi pris en tenaille entre la nécessité d'affirmer le bien fondé de la prescription et de la maîtrise de la technique, et l'expérience quotidienne de la «tricherie », des écarts à la norme.

De ce fait, la gestion de la production par l'encadrement de proximité se fait sur la base de l'acceptation de la dérive des pratiques de sécurité tant que les prises de risques n'aboutissent pas à un accident de travail. La remontée des informations venant des FE ne se fait pas de façon directe mais après lecture et traduction par les différents acteurs de la ligne hiérarchique; toutefois, le détail de la façon dont est utilisé l'indicateur de gestion par chaque acteur ne pourra être développé ici. Cependant, nous pouvons affirmer que la vision de la sécurité de chacun est guidée par les déterminants de son activité: par exemple, la responsabilité de l'encadrement de proximité quant au respect des objectifs de production, et la pression que cela représente, l'amène à fermer les yeux sur les prises de risque des opérateurs. De ce fait, l'usage des FE sera guidé par ces contraintes: elles peuvent être dans le cas de réunions de l'encadrement de proximité une aide à la prise de décision concernant la maintenance 
des machines, mais elles peuvent également être un élément de justification de certaines dépenses pour la direction de production demandées à la direction générale.

Ainsi, concernant la vision de la sécurité, trois situations peuvent être identifiées:

- celle de l'encadrement de proximité qui voit et connaît la situation mais n'a pas les ressources pour créer les conditions favorables pour travailler en sécurité et en productivité à la fois ;

- celle des cadres de production qui ne transmettent que ce qui est transcrit sur les FE soit uniquement les problèmes techniques et pas le problème de qualité des composants; pour autant notons que eux aussi voient comment les choses se passent dans la réalité mais ils sont soumis aux mêmes pressions que l'encadrement de proximité;

- celle de la direction qui ne reçoit qu'un résumé de la situation et donc pas d'informations en dehors des gros problèmes techniques nécessitant de lourdes dépenses ou pouvant justifier des retards de production.

En conclusion de ces premières observations, nous pouvons dire que les opérateurs réalisent des compromis pour faire face aux aléas. Ces compromis peuvent se faire au détriment de leur sécurité. En effet, ils sont amenés à prendre des risques, par exemple en intervenant sur la machine alors que celle-ci est en marche (ce qui génère des risques de coupure lorsque les interventions se font près des couteaux ou d'écrasements des doigts lorsqu'elles se font près des roulements) pour atteindre la production en terme de qualité, quantité et respect des délais, ce qui peut aboutir à des AT.

Lorsque le système tombe en panne, il bénéficie d'une maintenance spécialisée (changement de pièces...). En revanche, les « prises de risque » avec violation des règles de sécurité ne sont pas transmises dans les indicateurs. Les mesures de correction et de prévention en sont donc appauvries et cela a des conséquences sur les conditions de travail (en terme de sécurité notamment). De plus, au-delà de l'IG (indicateur de gestion) lui-même, les informations concernant les prises de risque ne sont pas discutées bien que connues d'une partie de l'encadrement. Les FE tendent à servir de protection pour l'encadrement de proximité pour éviter de discuter d'un problème auquel il ne sait pas répondre mais auquel il doit travailler puisque le prescrit et la hiérarchie l'exigent.

Pour expliquer ces effets, nous allons revenir sur les conséquences du recours aux indicateurs de gestion.

\section{3.- Recours aux indicateurs de gestion : quelles conséquences pour l'activité?}

Nous l'avons dit précédemment: un outil de gestion est utilisé par rapport à une activité, que ce soit celle de l'entreprise à un niveau " macro » (par exemple: part de marché) ou celle des opérateurs à un niveau « micro » (par exemple: taux d'absentéisme). Il constitue une vue synthétique de cette activité qui dans certains cas en donne une vision limitée. Toutefois, il est difficile d'évaluer des performances sans avoir recours à ce genre de données (Moisdon, 1997).

Concernant la sécurité au travail, des indicateurs sont bien sûr utilisés pour veiller à son évolution. Toutefois, ces indicateurs ne sont pas perçus comme des indicateurs de gestion en tant que tels mais sont plutôt traités de façon annexe. Cela peut être dû à certaines de leurs limites. Par exemple, les indicateurs de fréquence et de gravité des AT traditionnellement utilisés ne sont pas révélateurs de l'état des conditions de travail dans une entreprise. En outre, leur diminution ne permet pas nécessairement de conclure à une amélioration des conditions de travail en termes de santé et de sécurité au travail (SST) (Cadieux, Roy, \& Desmarais, 2004). Ils indiquent davantage les échecs que les succès, ils sont influencés par le hasard et ne permettent pas de prédire les performances futures. De plus, ils ne servent généralement qu'à indiquer le coût des dépenses notamment lorsqu'ils sont établis au niveau national. Cependant, si on prend le cas du nombre d'AT par exemple, cet indicateur a tout de 
même l'avantage de chiffrer la sécurité et donc, dans une certaine mesure, d'être considéré comme un indicateur de rentabilité (Gollac \& Volkoff, 2000).

Les indicateurs de gestion ne vont donc rendre compte qu'en partie de l'activité et de ses résultats. Ils sont davantage révélateurs de l'efficacité de l'entreprise, c'est-à-dire du fait qu'elle atteigne ou non les résultats sans prendre en compte l'efficience c'est-à-dire les moyens mis en œuvre pour y arriver.

Les indicateurs de gestion ne permettent pas d'expliquer le but de la prise de risque: ils expriment les résultats obtenus (produits en kilogramme, taux de déchets, de rebuts...) mais pas les compromis, d'où une incompréhension de la prise de risque chez l'encadrement et des tensions entre celui-ci et les opérateurs de la production.

En réalité, le recours systématique aux IG pour prendre des décisions va amener l'encadrement à fermer les yeux sur les situations à risque et surtout sur un phénomène sur lequel il n'a aucune marge de manœuvre pour agir. Nous avons présenté la vision de la sécurité de chaque acteur dans le chapitre précédent. Cependant, nous avons pu noter une évolution de cette vision et de l'usage des FE, suite à la présentation des résultats de l'intervention ergonomique effectuée en réponse à la demande concernant les accidents de travail. Il avait alors été mis en débat que le recours aux FE masquait les interventions sur machine en marche et que par conséquent le cœur du problème, c'est-à-dire la qualité des composants, ne pouvait être traité. De plus, il avait également été pointé que le management actuel ne permettait pas de comprendre la prise de risque car aucune place n'était laissée aux opérateurs pour justifier de leur comportement, seule la sanction était appliquée. Suite à cela, la direction a décidé de changer de «style de management » (selon ses termes) en instaurant des réunions chaque matin auxquelles participent les cadres de la maintenance et de la production. Lors de ces réunions, le directeur général a redéfini le rôle de l'encadrement de proximité au regard de la sécurité: il ne s'agit plus de sanctionner mais d'essayer de comprendre dans un dialogue avec les opérateurs pourquoi les prises de risque existent. Les FE ne servent plus de support aux réunions quotidiennes de l'encadrement de proximité, mais uniquement à programmer et gérer la maintenance des machines.

Cependant, dans les faits, cette évolution ne s'est pas encore traduite par les résultats escomptés, nous pensons qu'elle n'est qu'amorcée et qu'elle demande à être poursuivie. Aujourd'hui la direction connaît les problèmes et les considère comme importants, la ligne hiérarchique est informée de la même façon des situations à risque et de leurs causes, pourtant les solutions proposées ne portent pas sur la qualité des composants mais sur la sécurité « technique » (capotage machine, affichage...). On observe ainsi que l'information sur les situations à risque a en quelque sorte le droit de remonter dans la ligne hiérarchique, elle est discutée lors de réunions qui acquièrent un statut d'indicateur de gestion pour la direction mais les décisions ne sont pas prises. La question reste donc de savoir pourquoi ce blocage.

Le but de notre démarche sera à terme de permettre une articulation entre sécurité et production par un usage repensé des indicateurs (FE, réunions, indicateur sécurité...) amenant des prises de décision pour une action sur les causes profondes. Pour cela, nous pouvons dès lors proposer quelques avancées (auxquelles la suite de notre recherche pourra peut-être répondre): il s'agirait de travailler sur les causes des problèmes qualité pour éviter les interventions, en mobilisant les compétences des opérateurs en phase de diagnostic et de construction des solutions. Ainsi il deviendrait nécessaire de faire s'articuler un IG de production (qualité des composants concernant le passage de celles-ci dans la machine) et les IG de sécurité (le nombre de prises de risque par exemple). Ce travail d'articulation pourrait être ensuite reconnu dans la norme par la formalisation d'un processus.

\section{Conclusion/Discussion}

L'analyse ergonomique du travail a permis de mettre en évidence les compromis faits par les opérateurs pour atteindre les objectifs de la production. Ces compromis permettent d'obtenir les résultats 
demandés mais au prix de prises de risque qui parfois débouchent sur un accident. Les risques sont pris notamment parce que les contraintes de travail conduisent les opérateurs à effectuer des interventions sur machine en marche pour éviter à la fois les défauts qualité et l'augmentation du taux de rebuts mais également les retards de production.

L'analyse des feuilles d'équipes a quant à elle montré que ces interventions machine en marche n'apparaissent pas du tout. En effet, ne sont transcrits sur cet indicateur que les arrêts machines et donc ni la prise de risque ni ses causes. La prise de risque va donc masquer les problèmes qualité en évitant les arrêts et les décisionnaires n'en seront pas informés.

Ces deux analyses effectuées en parallèle nous ont amenés à constater que ce type d'indicateur de gestion ne permet pas de rendre compte de l'activité des opérateurs mais seulement des résultats de celle-ci. De ce fait, la prise de décision par l'encadrement de proximité sur la base de ces feuilles peut être non satisfaisante. Par exemple, les opérateurs réclament qu'un travail soit fait sur la qualité des composants (car elles sont les causes des interventions sur machine en marche). Mais puisque le seul moyen de communication avec les supérieurs est la feuille d'équipe et que cette feuille ne laisse pas apparaître ce type de problème qualité, le problème n'est pas traité. En réalité seuls sont traités les problèmes techniques, par exemple les remplacements de pièces.

On constate donc un décalage entre ce qui se joue dans la réalité du travail et la représentation qu'en a la hiérarchie par les indicateurs. Il nous faut ajouter que ces représentations sont surtout celles de l'encadrement éloigné (direction). En effet, les cadres de proximité ont exprimé au cours d'entretiens qu'ils comprenaient les compromis faits par les opérateurs, compromis qu'ils sont parfois amenés eux-mêmes à faire s'ils doivent intervenir (pour remplacer un absent par exemple). De plus, ils ont généralement commencé à travailler sur la chaîne de montage avant de monter les échelons hiérarchiques, et ont donc une certaine connaissance des contraintes de travail, ce qui n'est pas le cas de leurs supérieurs. De ce fait, les cadres de proximité sont pris dans un étau entre d'une part les contraintes remontant de l'activité des opérateurs (prise de risque) et les injonctions descendant de la hiérarchie (objectifs de production). Pour faire face à ces difficultés, et surtout au problème des situations à risque auquel ils ne peuvent répondre, ils vont utiliser les FE comme un bouclier:

- dans un premier temps: les informations concernant les prises de risque, soit les interventions sur machine, sont transmises mais les réponses de l'encadrement supérieur ne permettent pas de trouver des solutions;

- dans un second temps: du fait de ce blocage et puisque l'information n'est pas transmise par l'indicateur, le problème est occulté.

Il ne s'agit donc pas d'un défaut de l'IG (nous l'avons déjà dit, un IG ne constitue jamais qu'un « résumé » de la situation de travail) mais d'un choix de l'encadrement dû à un manque de marges de manœuvre ne lui permettant pas de traiter le problème de la qualité des composants. Pourtant, les négociations concernant les composants sont possibles et ont lieu mais lorsque c'est la production qui est touchée de façon directe (retard, défaut produit fini...), soit en changeant de fournisseur, soit en demandant au fournisseur d'améliorer les composants.

Les compromis faits par les opérateurs ont donc un coût sur la santé et la sécurité mais également sur les rapports sociaux qui se dégradent au fur et à mesure que les partenaires sont dans l'incompréhension mutuelle. On pourrait donc conclure qu'il faille enrichir les indicateurs de gestion pour les rendre plus représentatifs de la réalité et ainsi favoriser le dialogue. Il s'agirait alors ici de mettre en débat la réalité des situations de travail. Cependant, il ne faut pas perdre de vue que l'activité de gestion de ces indicateurs peut constituer une charge pour ceux qui les renseignent mais également pour ceux qui les traitent, charge d'autant plus importante que l'on y ajoute encore des données. Les cadres disent bien lors d'entretiens que l'important dans les indicateurs est leur facilité et leur rapidité de traitement. La solution ne semble donc pas être dans la modification du caractère quantitatif mais dans la nature des informations à transmettre. Les données issues de l'activité enrichiraient ainsi les indicateurs de gestion par la connaissance des compromis et donc des dysfonctionnements qualité. 
Pour autant, un travail sur la cause des problèmes, les aléas dus à la qualité des composants, reste indispensable.

L'intervention ergonomique et la présentation des résultats concernant les limites de l'indicateur mais aussi les causes des interventions ont permis la mise en débat de la situation de travail: les prises de risque ne doivent plus être perçues comme quelque chose d'inadmissible et de sanctionnable mais comme un événement dont il faut comprendre les causes pour y remédier et participant à l'obtention des résultats de production. Depuis, une nouvelle « politique sécurité » est affichée: lors de réunions quotidiennes (appelées réunions du matin), l'encadrement doit observer les interventions sur machine en marche et demander les explications aux opérateurs sur les raisons de ces prises de risque. On passe donc d'une vision orientée sur l'opérateur à une vision plus globale ciblée sur le système de travail. Cependant, cette nouvelle politique ne semble pas aller au bout de ce qu'elle affiche: en effet, même si les problèmes de qualité des composants sont exposés, ils ne sont pas résolus. En revanche, des bilans sur l'état des portes de sécurité (sont-elles shuntées ou non) sont faits et doivent être remédiés : on revient donc à une vision technique de la sécurité. De même, lorsqu'un AT a lieu, seules des réponses techniques sont apportées (capotage, affichage...).

Il est donc d'abord nécessaire de voir quelles sont les limites concrètes de l'indicateur, dans notre cas: l'absence de la prise en compte des problèmes qualité des composants générant des interventions sur machine en marche soit un IG en quelque sorte trop quantitatif. L'enrichir avec des données davantage qualitatives a permis une certaine prise de conscience, même si cela reste encore fragile : le dialogue a changé mais les actes n'ont pas encore suivi. De plus, suite à cette évolution, l'utilisation des FE est devenue plus annexe que précédemment: dans un premier temps, elles étaient l'instrument central des réunions quotidiennes de l'encadrement de proximité, aujourd'hui, elles sont utilisées uniquement pour prévoir les interventions de maintenance sur les machines. Les problèmes de qualité des composants sont abordés lors de ces réunions en lien avec la sécurité des opérateurs. Mais, ces derniers estiment que de toute façon la direction ne répond pas à leurs observations (notamment à cause du coût que cela engendrerait) et restent donc en quelque sorte « résignés », ne cherchant pas à faire remonter ces informations au-delà de ces réunions.

L'intégration de la sécurité (par un autre type de contrôle sur les composants) dans un processus concernant le choix des composants pourrait favoriser la transmission d'informations puisque cela deviendrait une obligation de la norme. Ainsi, l'entreprise se doterait de deux types d'IG:

- un indicateur de résultats qui permet de voir si ceux-ci ont été atteints ou non, comme la FE;

- un indicateur de levier ou suivi intégré dans un processus qui permet de connaître les évolutions tendancielles et anticiper en conséquence, cet IG porterait par exemple sur un déterminant de l'activité comme le nombre de bourrage rendant compte de la qualité du passage du composant.

Cela s'accompagnerait de la formalisation dans la norme afin que les décisions prises le soient par rapport à ces deux IG.

En revanche, les décisions prises en rapport avec ces deux IG devront se faire au niveau de la direction car c'est à ce niveau que les marges de manœuvre sont les plus importantes pour décider de changer ou non un composant.

Pour finir, la question sous-jacente qui se pose dans le contexte particulier d'un pays en voie de développement industriel et donc particulièrement exposé aux pressions de la mondialisation, est celle de comprendre comment se construit la qualité et comment elle met en situation la sécurité. Dans ce cas particulier, on voit que la qualité est avant tout un argument marketing (comme dans la plupart des entreprises mais avec une plus forte prégnance). Pour y répondre vite, la qualité n'a pas été réellement construite. Il y a donc une contradiction entre ce qui est affiché (politique qualité, politique sécurité...) et la réalité de la situation (les conditions d'échange avec les fournisseurs de composant notamment), ce qui va se répercuter sur les conditions de travail dans l'atelier de production. L'intervention de l'ergonome ne se situe donc plus seulement à ce niveau mais se déplace au niveau 
de la prise de décision de la direction (Ghram, Fournier, Khalfallah, Benchekroun \& Six, 2009), là où va se jouer la construction de la qualité du produit mais également du processus. Il serait également intéressant de s'assurer que les fournisseurs de composants, au demeurant presque tous européens comme nous l'avons déjà indiqué et fournisseurs de sites de production européens, n'opèrent pas de sélections dans leurs livraisons aux différents sites. La réponse à une telle question, qui est loin d'être facile à obtenir, serait intéressante dans la perspective du management de la qualité (Benchekroun, Ghram, Fournier, Six, \& Akrout, 2009). En effet, soit les produits livrés sont de moindre qualité et alors on aurait là une origine possible des problèmes rencontrés par l'entreprise et analysés dans cette recherche, soit les produits livrés sont de qualité attendue et alors l'origine des problèmes est à rechercher ailleurs, par exemple dans l'état des machines, leur maintenance ou encore la connaissance que les opérateurs ont du processus.

\section{RÉFÉRENCES}

AFNOR (2001). Qualité et systèmes de management ISO 9000. Paris: AFNOR.

Amalberti, R. (2004). De la gestion des erreurs à la gestion des risques. In P. Falzon (Ed.), Ergonomie (pp. 285-300). Paris: Presses Universitaires de France.

Ben Hammouda, H. (1995). Tunisie, ajustement et difficulté de l'insertion internationale. Paris: L'Harmattan.

Benchekroun, T.H., Ghram, R., Fournier, C., Six, F., \& Akrout, M. (2009). Condiciones para un desarrollo sustentable de sistemas de trabajo en los países emergentes: El caso de tres empresas en Túnez. Laboreal, $5(1), 70-83$.

Berry, M. (1983). Une technologie invisible? L'impact des instruments de gestion sur l'évolution des systèmes humains. Paris: Editions de l'Ecole Polytechnique.

Bourgeois, F., \& Hubault, F. (2005). Prévenir les TMS. De la biomécanique à la revalorisation du travail, l'analyse du geste dans toutes ses dimensions. @ctivités, 2(1), 19-36, http://www.activites.org/v2n1/ bourgeois.pdf

Bourgeois, F., Lemarchand, C., Hubault, F., Brun, C., Polin, A., \& Faucheux, J.M. (2000). Troubles musculosquelettiques et travail, quand la santé interroge l'organisation. Lyon: ANACT.

Bourrier, M. (2005). The contribution of organizational design to safety. European Management Journal, 23(1), 98-104.

Brandenburg, H., \& Wojtyna, J-P. (2006). L'approche processus, mode d'emploi. Paris: Edition d'Organisation.

Cadieux, J., Roy, M., \& Desmarais, L. (2004). Changement de perspective dans la mesure de performance en SST. Actes du $15^{\text {ème }}$ congrès de l'AGRH, pp. 2583-2592

Campinos-Dubernet, M, \& Jougleux, M. (2003). L'assurance qualité: quelles contributions à la qualité des services? Revue française de gestion, 5(146), 81 - 98.

Campinos-Dubernet, M., \& Marquette, C. (1997). Les normes d'assurance qualité ISO 9000 : une opportunité de rationalisation des processus d'apprentissage de l'entreprise ? Cahiers de recherche $d u$ Groupement d'Intérêt Public "Mutations industrielles", 60, 2-46.

Cheikh S., (2000). Certification Qualité et Conditions de travail. Actes du 34 ème congrès de la SELF, pp. 8187.

Coutarel, F., Daniellou, F., \& Dugué, B. (2005). La prévention des troubles musculo-squelettiques: quelques enjeux épistémologiques. @ctivités, 2(1),3-18, http://www.activites.org/v2n1/coutarel.pdf

De La Garza, C., \& Fadier, E. (2004). Sécurité et prévention : repères juridiques et ergonomiques. In P. Falzon (Ed.), Ergonomie (pp. 159-174). Paris: Presses Universitaires de France.

Dionne-Proulx, J., Carrière, J-B., \& Beauchamp, Y. (2005). La certification ISO et la SST: Étude empirique et résultats terrain. PISTES, 7(1), 1-13, http://www.pistes.uqam.ca/v7n1/pdf/v7n1a3.pdf 
Faverge, J-M. (1970). L'homme agent d'infiabilité et de fiabilité du processus industriel. Ergonomics, 13(3), 301-327.

Forrierre, J. (2008). La prévention du risque routier professionnel par l'analyse de l'activité : le cas des conducteurs de travaux des travaux publics. Thèse de Doctorat en Ergonomie, Université Charles-deGaulle - Lille 3, Lille, France.

Garrigou, A., Peeters, S., Jackson, M., Sagory, P., \& Carballeda, G. (2004). Apports de l'ergonomie à la prévention des risques professionnels. In P. Falzon (Ed.), Ergonomie (pp. 497-514). Paris: Presses Universitaires de France.

Ghram, R., Fournier, C., Khalfallah, T., Benchekroun T-H., \& Six, F. (2009). Strategic decision making: work analysis of a company manager. Paper presented at the 17th World Congress on Ergonomics, Beijing, China.

Goguelin, P. (2003). Risque et prise de risque : les concepts. In P. Goguelin, \& X. Cuny (Eds.), La prise de risque dans le travail: actes d'études et de réflexion (pp. 19-36). Toulouse: Octarès.

Gollac, M., \& Volkoff, S. (2000). Les conditions de travail. Tournai: La Découverte.

Guérin, F., Laville, A., Daniellou, F., Duraffourg, J., \& Kergueken, A. (2001). Comprendre le travail pour le transformer. Lyon: Editions de l'ANACT.

Hoc, J-M. (2004). La gestion de situation dynamique. In P. Falzon (Ed.), Ergonomie (pp. 517-531). Paris: Presses Universitaires de France.

Hubault, F. (1998). Pour une ergonomie de l'encadrement. Performances Humaines et Techniques, $n^{\circ}$ Hors Série Ergonomie de l'encadrement - pouvoirs et responsabilités des cadres, Séminaire Paris 1, Toulouse, 2-9.

Iazykoff, V. (2004). L'intégration organisationnelle de la certification. Le cas de deux divisions régionales d'une grande entreprise. Revue française de gestion, 5(152), 97-114.

Laurens, P. (1997). Qualité et communication organisationnelle. Thèse de doctorat en sciences de l'information et de la communication, Université Toulouse 2, Toulouse, France.

Leplat, J. (1997). Regards sur l'activité en situation de travail: contribution à la psychologie ergonomique. Paris: PUF.

Leplat, J. (1998). About implementation of safety rules. Safety science, 29, 189-204.

Lérat-Pytlak, J. (2002). Le passage d'une certification ISO 9001 à un management par la qualité totale. Thèse de Doctorat en Sciences de Gestion, Université des Sciences Sociales - Toulouse 1, Toulouse, France.

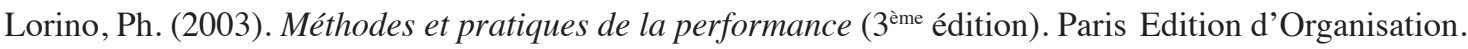

Lorino, Ph. (2009). Concevoir l'activité collective conjointe : l'enquête dialogique. Étude de cas sur la sécurité dans l'industrie du bâtiment. @ ctivités 6(1), 87-110, http://www.activites.org/v6n1/v6n1.pdf

Kerbal, A. (2000). Ergonomie et critères de gestion des entreprises. Actes du $34^{\text {ème }}$ congrès de la SELF, pp. 12-18.

Mambi-El-Sendegele, F. (2001). Règles - normes. Certification de qualité et management industriel: le cas des organismes de formation. INNOVATIONS, 1(13), 133-158.

Mispelblom, F. (1995). Au delà de la qualité : démarches qualité, conditions de travail et politiques du bonheur. Paris: Syros.

Moisdon, J.C. (1997). Du mode d'existence des outils de gestion. Gap: Seli Arslan.

Reason, J. (193). L'erreur humaine (trad. J.-M. Hoc). Paris: Presses Universitaires de France.

Sauvagnac, C., \& Falzon, P. (2000). Gestion des savoirs et politique qualité: le rôle de l'ergonomie. Actes du $34^{\text {ème }}$ congrès de la SELF, pp. 104-115. 
Savall, H., \& Zardet, V. (2003). Maîtriser les coûts et les performances cachées (4 ${ }^{\mathrm{ème}}$ édition). Paris: Economica.

Sfeir, A. (2006). Tunisie, Terre de paradoxe. Paris: L'Archipel.

Six, F. (1999). De la prescription à la préparation du travail, apports de l'ergonomie à la prévention et à l'organisation du travail sur les chantiers du BTP. Habilitation à Diriger la Recherche, Université Charles De Gaulle - Lille 3, Lille, France.

Teiger, C. (1993). Représentation du travail, travail de la représentation. In A. Weill-Fassina, P. Rabardel, \& D. Dubois (Eds), Représentations pour l'action (pp. 311-344). Toulouse: Octarès.

Terssac, G. de (1994). L'implicite et le décrit. De l'organisation des systèmes de production. Projet, 236, 1622.

\section{RÉSUMÉ :}

Les préoccupations des sociétés relèvent prioritairement des questions de rentabilité. Pour pérenniser leurs activités, la majorité fait le choix de la certification. Cette démarche requiert alors l'usage d'indicateurs. Certaines études ont montré en quoi cette démarche peut parfois faire « oublier » les questions de sécurité.

Notre recherche s'est déroulée dans une entreprise basée en Tunisie. Celleci a mis en œuvre depuis 2004 un système de management de la qualité qui a débouché sur une certification. La démarche a été initiée pour répondre aux demandes des clients et pour pérenniser l'activité de l'entreprise.

Nous montrerons ici en quoi le recours à des indicateurs peut parfois éloigner les questions de sécurité. Ce problème de décalage entre ce qui ressort de l'indicateur concernant l'activité de travail et ce qui se passe réellement dans la situation de travail amène à des prises de décision qui ne permettent pas l'amélioration des conditions de travail.

MOTS-CLÉS

indicateurs de gestion, certification, sécurité, compromis, activité

\section{RÉFÉRENCEMENT}

Fournier, C., Ghram, R., Benchekroun, T.-H., \& Six, F. (2011). Tension entre indicateurs de production et indicateurs de sécurité : Le cas de la certification d'une entreprise tunisienne. Activités, 8(1), pp. 44-61, http://www.activites.org/v8n1/v8n1.pdf

Article soumis le 12 juin 2009, accepté pour publication le 22 octobre 2010 